\title{
Single Breath-Hold Non-Contrast Thoracic MRA Using Highly- Accelerated Parallel Imaging With a 32-element Coil Array
}

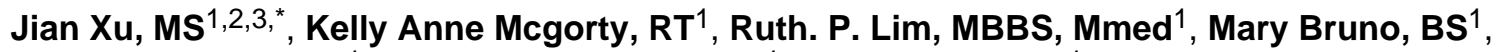 \\ James S. Babb, $\mathrm{PhD}^{1}$, Monvadi B. Srichai, $\mathbf{M D}^{1}$, Daniel Kim, $\mathbf{P h D}^{1}$, and Daniel K. \\ Sodickson, MD, $\mathrm{PhD}^{1}$ \\ ${ }^{1}$ Bernard and Irene Schwartz Center for Biomedical Imaging, Department of Radiology, New York \\ University School of Medicine, New York, NY, USA \\ ${ }^{2}$ PolyTechnic Institute of New York University, Brooklyn, New York, NY, USA \\ ${ }^{3}$ Siemens Medical Solutions USA Inc., New York, NY, USA
}

\begin{abstract}
OBJECTIVE-To evaluate the feasibility of performing single breath-hold 3D thoracic noncontrast magnetic resonance angiography (NC-MRA) using highly-accelerated parallel imaging.

MATERIALS AND METHODS-We developed a single breath-hold NC MRA pulse sequence using balanced steady state free precession (SSFP) readout and highly-accelerated parallel imaging. In 17 subjects, highly-accelerated non-contrast MRA was compared against electrocardiogram (ECG)-triggered contrast-enhanced MRA. Anonymized images were randomized for blinded review by two independent readers for image quality, artifact severity in 8 defined vessel segments and aortic dimensions in 6 standard sites. NC-MRA and CE-MRA were compared in terms of these measures using paired sample $t$ and Wilcoxon tests.
\end{abstract}

RESULTS-The overall image quality ( $3.21 \pm 0.68$ for NC-MRA vs. $3.12 \pm 0.71$ for CE-MRA) and artifact $(2.87 \pm 1.01$ for NC-MRA vs. $2.92 \pm 0.87$ for CE-MRA) scores were not significantly different, but there were significant differences for the great vessel and coronary artery origins. NC-MRA demonstrated significantly lower aortic diameter measurements compared to CE-MRA; however, this difference was not considered clinically relevant (>3 mm difference) for less than $12 \%$ of segments, most commonly at the sinotubular junction. Mean total scan time was significantly lower for NC-MRA compared to CE-MRA (18.2 \pm 6.0 s vs. $28.1 \pm 5.4 \mathrm{~s}$, respectively; $\mathrm{p}<0.05)$.

CONCLUSION-Single breath-hold NC-MRA is feasible and can be a useful alternative for evaluation and follow-up of thoracic aortic diseases.

\section{Keywords}

Thoracic MRA; Non Contrast; Breath-hold; Parallel Imaging

\section{INTRODUCTION}

Contrast-enhanced 3D magnetic resonance angiography (MRA) is routinely used to diagnose aortic disease because of its high spatial resolution, reliability and potential for multi-planar assessment (1-3). However, for thoracic MRA, visualization of the aortic root

Corresponding author: Jian Xu, MS, Department of Radiology, New York University School of Medicine, 660 First AVE, New York, NY 10016, Phone:01- 212-263-4831, Fax: 01-212-263-4830, jian.xu.sz@gmail.com. 
and ascending aorta is often hindered by cardiac motion, and orthogonal aortic dimensions cannot be accurately measured. Electrocardiographic (ECG)-triggered 3D contrast-enhanced MRA (CE-MRA) of the thoracic aorta can improve vessel wall sharpness, resulting in higher quality images, particularly of the aortic root and more accurate diagnosis of disease (4-7). However, the data acquisition window per cardiac cycle needs to be minimized to avoid cardiac motion artifacts, and this decreases imaging efficiency, imposing limits on spatial resolution or volumetric coverage for breath-hold $(\mathrm{BH})$ thoracic MRA. In addition, CE-MRA relies on accurate timing of contrast bolus arrival during acquisition of the center of k-space. In bradycardic patients, acquisition time may become inordinately long, leading to respiratory motion, subtraction misregistration, suboptimal arterial contrast, as well as venous enhancement. In addition, patients with impaired renal function are at risk of nephrogenic systemic fibrosis (8) associated with gadolinium-based contrast agents (9-11) with CE-MRA. Therefore, a non-contrast MRA technique of comparable quality and reliability as CE-MRA is highly desirable.

Navigator-gated, ECG-triggered 3D balanced steady state free precession (b-SSFP) MRA with T2 preparation and fat suppression is widely used for coronary MRA (12-14), because of its high signal-to-noise ratio (SNR) and contrast-to-noise ratio (CNR). This technique has been used for thoracic MRA (6-7,15-16), but scan time is on the order of 4-10 minutes. Modern MRI systems equipped with a 32-element coil array offer the potential for baseline SNR improvements over traditional coil arrays with fewer elements, and are capable of multidimensional parallel imaging (17-19) with reduced noise amplification and preserved SNR compared to 1D accelerations (20). Highly accelerated parallel imaging can be used to shorten BH duration and/or improve spatiotemporal resolution. The feasibility of ECGtriggered BH MRA of thoracic aorta using an accelerated 3D b-SSFP was first demonstrated in volunteers by Niendorf et al. with an acceleration factor 2 and BH duration of 40 heartbeats (21).

We have developed a single BH ECG-triggered MRA pulse sequence using a 32-element coil array enabling two-dimensional image acceleration and acquisition of both the coil sensitivity and MRA data within the same heart beats at different cardiac phases (early systole and mid diastole, respectively) (22). Simultaneous acquisition of the coil sensitivity and MRA data minimizes registration errors which occur when they are acquired in separate breath holds (23). The purpose of this study was to evaluate the feasibility of performing single breath-hold NC-MRA with isotropic spatial resolution using highly-accelerated parallel imaging and to compare it with ECG-triggered CE-MRA for the assessment of the thoracic aorta in human subjects.

\section{MATERIALS AND METHODS}

This study protocol was HIPAA compliant and approved by our Institutional Review Board; all subjects provided written informed consent. We prospectively enrolled 17 subjects ( 7 healthy subjects, 10 patients, 13 males (mean age 39 \pm 18.5 years; range 23-79 years)) to undergo thoracic aorta MRA with a 1.5-T whole-body MRI system (MAGNETOM Avanto, Siemens AG, Germany) using a 32-element cardiac coil array (Invivo, USA). For evaluation of thoracic aortic disease, NC-MRA followed by CE-MRA sequences were performed in all subjects.

\section{Imaging Technique}

Imaging parameters for BH NC-MRA using b-SSFP were: TR/TE 2.3/1.6ms, flip angle $70^{\circ}$, FOV $300 \times 400 \mathrm{~mm}^{2}$, Matrix $192 \times 256$, slice number 40 52, A-P phase encode direction, true voxel size $1.6 \times 1.6 \times 1.6 \mathrm{~mm}^{3}$, bandwidth $500 \mathrm{~Hz} /$ pixel, $\mathrm{k}$-space lines per cardiac cycle $48, \mathrm{~T} 2$ preparation pulse with preparation time $40 \mathrm{~ms}$, fat suppression pulse, 2D parallel imaging 
with a generalized auto calibrating partial parallel acquisition (GRAPPA)(19) acceleration of 3 in the phase encode direction and 2 in the partition direction, 6/8 partial Fourier in both phase encode and partition directions, and partition oversampling $20 \%$. Both coil sensitivity (early systole) and MRA (mid diastole) data were acquired in the same BH, as shown in Figure 1.

Pre- and post-contrast ECG-triggered CE-MRA using a 3D gradient-echo fast low-angle shot (FLASH) pulse sequence was performed with similar parameters as NC-MRA to achieve the same spatial resolution: TR/TE $3.5 / 1.1 \mathrm{~ms}$, flip angle $17^{\circ}$, FOV $300 \times 400 \mathrm{~mm}^{2}$, Matrix 192×256, slice number 40 52, A-P phase encode direction, true voxel size $1.6 \times 1.6 \times 1.6 \mathrm{~mm}^{3}$, bandwidth $330 \mathrm{~Hz} /$ pixel, 1D GRAPPA acceleration factor $2,6 / 8$ partial Fourier in both phase encode and partition directions. Before acquisition of the post-contrast CE-MRA data set, a pre-contrast 3D FLASH image set was acquired as a mask and subtracted from the contrast-enhanced 3D FLASH data set to eliminate background signal in case the subtracted images needed. A standard test-bolus acquisition, using 1-ml of contrast material (Gd-DTPA, Magnevist, Berlex, Schering AG), was performed to determine the contrast arrival time in the ascending thoracic aorta. Post-contrast 3D CE-MRA was then performed under suspended respiration following administration of Gd-DTPA $0.15 \mathrm{mmol} / \mathrm{kg}$ at $2 \mathrm{cc} / \mathrm{sec}$ followed by $20 \mathrm{cc}$ saline flush at the same rate with image acquisition timed based on timing bolus (24). Image acquisition time was recorded for each patient for both CEMRA and BH NC-MRA.

\section{Image Analysis}

For both techniques, source images were anonymized and randomized for blinded review, by a cardiologist and a radiologist (with 8 and 5 years' experience respectively) for image quality, artifacts and pathology in 8 vessel segments (sinuses of Valsalva, sinotubular junction, ascending aorta, aortic arch, descending aorta, diaphragmatic aorta, coronary artery origins and great vessel origins), as shown in Figure 2. Images were reviewed on a 3D workstation capable of multi-planar reformation, maximum intensity projection and volume rendering (Leonardo, Siemens Healthcare). Image quality was graded on a 5 point scale: $0=$ non-diagnostic, $1=$ poor, $2=$ fair, $3=$ good, $4=$ excellent, and artifact severity was graded on a 5 point scale: $0=$ non-diagnostic, $1=$ severely limiting, $2=$ mildly limiting, $3=$ not limiting, $4=$ no artifact. Aortic dimensions outer wall to outer wall were measured using bi-orthogonal planes for each sequence at 6 standard sites (sinuses of Valsalva, sinotubular junction, ascending aorta at right pulmonary artery level, arch proximal to the left subclavian artery, descending aorta at left pulmonary artery level and diaphragmatic aorta).

\section{Statistical Analysis}

All results are summarized as mean \pm standard deviation (SD). CE-MRA and NC-MRA were compared with respect to qualitative ordinal image quality and artifact severity scores using the Wilcoxon matched-pairs signed ranked test, and a paired-sample t-test was used to compare the two MRA techniques in terms of scan time and aortic diameter at each anatomical site, with the mean of the 2 orthogonal measurements used for statistical analysis. The mean paired difference and $95 \%$ confidence interval (CI) were calculated for the true mean difference between methods in terms of diameter measures averaged over all subjects. The percentage and number of times the absolute value of the difference exceeded the threshold value of $3 \mathrm{~mm}$ was also calculated to determine if the difference was clinically relevant (1,25-27). Bland-Altman analysis was used to compare the aortic dimensions measured from the CE-MRA and NC-MRA. SPSS (v.13, Chicago IL) was used for all tests and statistical significance was defined as $\mathrm{p}<0.05$ level. 


\section{RESULTS}

Of 17 subjects studied, mean total scan time was significantly lower for NC-MRA compared to CE-MRA ( $18.2 \pm 6.0$ s vs. $28.1 \pm 5.4 \mathrm{~s}$, respectively; $\mathrm{p}<0.05)$. In the 10 patients studied, CE-MRA diagnosed 5 aneurysms, 2 dilated aortic roots, 1 coarctation, 1 aortic valve replacement and 1 normal. NC-MRA identified the same pathology and was in agreement for all subjects. Typical images are shown in Figure 3 and 4.

In total, 136 vascular segments were scored for image quality and artifacts for the 17 subjects imaged with each technique. Image quality and artifact severity scores are summarized in Table 1. Overall 16 segments from both readers for CE-MRA and 13 segments from both readers for NC-MRA were considered non-diagnostic or degraded by severe artifact. There was no significant difference in overall image quality $(3.21 \pm 0.68 \mathrm{NC}$ MRA vs. $3.12 \pm 0.71$ for CE-MRA, respectively; $\mathrm{p}=0.145$ ) and overall artifact scores (2.87 \pm 1.01 NC-MRA vs. $2.92 \pm 0.87$ for CE-MRA, respectively; $\mathrm{p}=0.287$ ) between the 2 techniques for all evaluated thoracic aortic segments. There were statistically significant differences in image quality in the great vessel origins ( $3.58 \pm 0.49$ for CE-MRA vs. $2.39 \pm 0.78$ for NC-MRA, respectively; $\mathrm{p}=0.0001$ ), where signal loss due to susceptibility with b-SSFP readout was noted as shown in Figure 3, and coronary artery origins $(1.53 \pm 0.50$ for CE-MRA vs. $2.31 \pm 0.93$ for NC-MRA, respectively; $p=0.013$ ) where increased motion artifact was noted for CE-MRA compared to NC-MRA. The difference was not statistically significant (2.86 \pm 0.56 for CE-MRA vs. $3.11 \pm 0.65$ for NC-MRA, respectively; $p>0.05)$ at the level of aortic root.

In total, 102 segments were measured for aortic diameter for each technique. Mean aortic dimensions from CE-MRA and NC-MRA images are summarized in Table 2. Overall, there was a systematic bias for lower dimension measurements with NC-MRA compared to CEMRA. Clinically relevant differences ( $>10 \%$ difference) were noted for less than $12 \%$ of segments, most commonly at the sinotubular junction. Bland-Altman analysis (Figure 5) revealed a overall mean difference (NC-MRA minus CE-MRA) in aortic diameter between the two techniques of $-0.073 \pm 0.144 \mathrm{~cm}$, and upper and lower $95 \%$ limits of agreement were $0.210 \mathrm{~cm}$ and $-0.357 \mathrm{~cm}$, respectively.

\section{DISCUSSION}

In this study, we evaluated a single breath-hold NC-MRA against CE-MRA of the thoracic aorta, and demonstrated the feasibility of performing highly accelerated single BH NCMRA with isotropic spatial resolution and diagnostic image quality. Compared with ECGtriggered CE-MRA, NC-MRA has potential benefits of shorter scan time and repeatability without the need for exogenous contrast agent, providing rapid, safe and entirely noninvasive assessment of the thoracic aorta with similar diagnostic image quality of the readers.

In 17 subjects, we found no significant difference in overall aortic image quality. At the segmental level, NC-MRA compared to CE-MRA demonstrated significantly superior image quality for visualizing the coronary artery origins but inferior image quality for visualizing the great vessel origins. NC-MRA demonstrated significantly lower aortic diameter measurements compared to CE-MRA; however, this difference was considered clinically relevant ( $>3 \mathrm{~mm}$ difference) for less than $12 \%$ of segments, most commonly at the sinotubular junction.

Compared to non-gated CE-MRA, ECG-triggered 3D CE-MRA of the thoracic aorta can improve visualization of the ascending aorta and aortic root (4), but is challenging due to competing demands of high spatial resolution and high temporal resolution within a breath- 
hold. In addition, its performance relies on accurate timing of contrast bolus arrival during the acquisition of the center of k-space. Navigator-gated and ECG-gated NC-MRA has been shown to provide better visualization of the aortic root, ascending aorta, and coronary arteries, compared with CE-MRA, but with significantly longer imaging times (16). The approach used in this study was able to provide diagnostic image quality (22) within a breath-hold, without gadolinium contrast. The relative advantage of CE-MRA is arguably superior vascular delineation due to the contrast enhancement. Conversely, due to its shorter acquisition window (110 ms vs. $216 \mathrm{~ms}$; NC-MRA vs. CE-MRA, respectively, p<0.05) and shorter overall acquisition time which minimize effects of cardiac and respiratory motion respectively, single BH ECG-triggered NC-MRA is relatively less susceptibile to motion artifact.

Of note, the difference in aortic diameter measurement at the sinotubular junction segment was relatively high $(23.5 \%$ with $>3 \mathrm{~mm}$ difference). This was probably caused by use of different trigger delay time or the difference in acquisition window per cardiac cycle. Outer wall to outer wall measurements using CE-MRA were challenging due to relatively low contrast between the arterial wall and surrounding fat with high flip angle T1 GRE imaging. This may have led to systematic measurement error at CE MRA, and could explain why NC-MRA measurements were smaller.

A major limitation of NC-MRA is its sensitivity to off-resonance (e.g., signal loss of great vessel origins) (28). Local shimming of the ascending aorta and arch region has been reported to be useful (6). Another limitation of this study is that no comparison was made with other angiographic methods such as digital subtraction angiography or computed tomography. Also due to the nature of b-SSFP weighting (29), bright venous signal was present at NC-MRA. However, because of the large caliber of the thoracic aorta and central veins, this did not impact readers' image quality assessments as evidenced by similar image quality scores for NC-MRA and CE-MRA. Finally, our preliminary investigation had a relatively small number of patients with a limited variety of pathology and sequences were always performed in the same order (non-randomized) to ensure that NC-MRA was truly non-contrast enhanced.

Planned work includes minimizing off-resonance artifact using local shimming, and a comparison between ECG-gated CE-MRA and NC-MRA using the same (2D) acceleration factors. We also plan further assessment of discrepancies in vessel caliber between CEMRA and NC-MRA with phantom testing.

In conclusion, highly-accelerated NC-MRA with b-SSFP is a highly promising method for rapid, noninvasive assessment of thoracic aorta diseases, especially in patients with poor IV access or contraindications to gadolinium contrast. Further experience in a larger clinical population would be invaluable to assess its clinical applicability and accuracy.

\section{Acknowledgments}

The authors would like to thank Thoralf Niendorf, Xiaoming Bi, Xiaodong Zhong and Zhongwei Zhang for their discussions, comments and help in the preparation of this manuscript. The authors would like to thank Ding Xia and $\mathrm{Li}$ Feng for providing some of the pictures and figures.

Grant Sponsors:

National Institutes of Health: R01-EB000447; American Heart Association: 0730143N. 


\section{References}

1. Prince MR, Narasimham DL, Jacoby WT, et al. Three-dimensional gadolinium-enhanced MR angiography of the thoracic aorta. AJR Am J Roentgenol. 1996; 166(6):1387-1397. [PubMed: 8633452]

2. Krinsky GA, Reuss PM, Lee VS, Carbognin G, Rofsky NM. Thoracic aorta: comparison of singledose breath-hold and double-dose non-breath-hold gadolinium-enhanced three-dimensional MR angiography. AJR Am J Roentgenol. 1999; 173(1):145-150. [PubMed: 10397116]

3. Gebker R, Gomaa O, Schnackenburg B, Rebakowski J, Fleck E, Nagel E. Comparison of different MRI techniques for the assessment of thoracic aortic pathology: 3D contrast enhanced MR angiography, turbo spin echo and balanced steady state free precession. Int J Cardiovasc Imaging. 2007; 23(6):747-756. [PubMed: 17285264]

4. Groves EM, Bireley W, Dill K, Carroll TJ, Carr JC. Quantitative analysis of ECG-gated highresolution contrast-enhanced MR angiography of the thoracic aorta. AJR Am J Roentgenol. 2007; 188(2):522-528. [PubMed: 17242264]

5. Bireley WR 2nd, Diniz LO, Groves EM, Dill K, Carroll TJ, Carr JC. Orthogonal measurement of thoracic aorta luminal diameter using ECG-gated high-resolution contrast-enhanced MR angiography. J Magn Reson Imaging. 2007; 26(6):1480-1485. [PubMed: 17968882]

6. Srichai MB, Kim S, Axel L, Babb J, Hecht EM. Non-gadolinium-enhanced 3-dimensional magnetic resonance angiography for the evaluation of thoracic aortic disease: a preliminary experience. Tex Heart Inst J. 2010; 37(1):58-65. [PubMed: 20200628]

7. Krishnam MS, Tomasian A, Malik S, Desphande V, Laub G, Ruehm SG. Image quality and diagnostic accuracy of unenhanced SSFP MR angiography compared with conventional contrastenhanced MR angiography for the assessment of thoracic aortic diseases. Eur Radiol. 2010; 20(6): 1311-1320. [PubMed: 20013276]

8. Kramer J, Moeller EL, Hachey A, Mansfield KG, Wachtman LM. Differential expression of GLUT2 in pancreatic islets and kidneys of New and Old World nonhuman primates. Am J Physiol Regul Integr Comp Physiol. 2009; 296(3):R786-793. [PubMed: 19073904]

9. Othersen JB, Maize JC, Woolson RF, Budisavljevic MN. Nephrogenic systemic fibrosis after exposure to gadolinium in patients with renal failure. Nephrol Dial Transplant. 2007; 22(11):31793185. [PubMed: 17890246]

10. Broome DR, Girguis MS, Baron PW, Cottrell AC, Kjellin I, Kirk GA. Gadodiamide-associated nephrogenic systemic fibrosis: why radiologists should be concerned. AJR Am J Roentgenol. 2007; 188(2):586-592. [PubMed: 17242272]

11. Kanal E, Broome DR, Martin DR, Thomsen HS. Response to the FDA's May 23, 2007, nephrogenic systemic fibrosis update. Radiology. 2008; 246(1):11-14. [PubMed: 17855656]

12. Bi X, Deshpande V, Carr J, Li D. Coronary artery magnetic resonance angiography (MRA): a comparison between the whole-heart and volume-targeted methods using a T2-prepared SSFP sequence. J Cardiovasc Magn Reson. 2006; 8(5):703-707. [PubMed: 16891229]

13. Shea SM, Deshpande VS, Chung YC, Li D. Three-dimensional true-FISP imaging of the coronary arteries: improved contrast with T2-preparation. J Magn Reson Imaging. 2002; 15(5):597-602. [PubMed: 11997902]

14. Niendorf T, Hardy CJ, Giaquinto RO, et al. Toward single breath-hold wholeheart coverage coronary MRA using highly accelerated parallel imaging with a 32-channel MR system. Magn Reson Med. 2006; 56(1):167-176. [PubMed: 16755538]

15. Francois CJ, Tuite D, Deshpande V, Jerecic R, Weale P, Carr JC. Unenhanced MR angiography of the thoracic aorta: initial clinical evaluation. AJR Am J Roentgenol. 2008; 190(4):902-906. [PubMed: 18356435]

16. Amano Y, Takahama K, Kumita S. Non-contrast-enhanced MR angiography of the thoracic aorta using cardiac and navigator-gated magnetization-prepared three-dimensional steady-state free precession. J Magn Reson Imaging. 2008; 27(3):504-509. [PubMed: 18307199]

17. Sodickson DK, Manning WJ. Simultaneous acquisition of spatial harmonics (SMASH): fast imaging with radiofrequency coil arrays. Magn Reson Med. 1997; 38(4):591-603. [PubMed: 9324327] 
18. Pruessmann KP, Weiger M, Scheidegger MB, Boesiger P. SENSE: sensitivity encoding for fast MRI. Magn Reson Med. 1999; 42(5):952-962. [PubMed: 10542355]

19. Griswold MA, Jakob PM, Heidemann RM, et al. Generalized autocalibrating partially parallel acquisitions (GRAPPA). Magn Reson Med. 2002; 47(6):1202-1210. [PubMed: 12111967]

20. Zhu Y, Hardy CJ, Sodickson DK, et al. Highly parallel volumetric imaging with a 32-element RF coil array. Magn Reson Med. 2004; 52(4):869-877. [PubMed: 15389961]

21. Niendorf, T.; McKenzie, C.; Spencer, M.; Farrar, N.; Dennis, ZR.; Rofsky, NM. Image quality improvements in whole body MRA of the aorta by empolying accelerated, non-contrast enhanced, cardiac gated 3D SSFP. Proc ISMRM 2004; Kyoto, Japan. 2004. p. 2570

22. Xu J, et al. A New Approach for Single breath-hold Whole Heart Coronary MRA Using HighlyAccelerated Parallel Imaging With a 32-element Coil Array. ISMRM. 2009

23. Kuhara S, Ninomiya A, Okada T, Kanao S, Kamae T, Togashi K. Whole-heart magnetic resonance coronary angiography with multiple breath-holds and automatic breathing-level tracking. J Appl Phys. 2010; 107(9)

24. Earls JP, Rofsky NM, DeCorato DR, Krinsky GA, Weinreb JC. Breath-hold single-dose gadolinium-enhanced three-dimensional MR aortography: usefulness of a timing examination and MR power injector. Radiology. 1996; 201(3):705-710. [PubMed: 8939219]

25. Potthast S, Mitsumori L, Stanescu LA, et al. Measuring aortic diameter with different MR techniques: comparison of three-dimensional (3D) navigated steady-state free-precession (SSFP), 3D contrast-enhanced magnetic resonance angiography (CE-MRA), 2D T2 black blood, and 2D cine SSFP. J Magn Reson Imaging. 2010; 31(1):177-184. [PubMed: 20027585]

26. Miao C, Chen S, Macedo R, et al. Positive remodeling of the coronary arteries detected by magnetic resonance imaging in an asymptomatic population: MESA (Multi-Ethnic Study of Atherosclerosis). J Am Coll Cardiol. 2009; 53(18):1708-1715. [PubMed: 19406347]

27. Hoogeveen RM, Bakker CJ, Viergever MA. Limits to the accuracy of vessel diameter measurement in MR angiography. J Magn Reson Imaging. 1998; 8(6):1228-1235. [PubMed: 9848733]

28. Axel L, Kolman L, Charafeddine R, Hwang SN, Stolpen AH. Origin of a signal intensity loss artifact in fat-saturation MR imaging. Radiology. 2000; 217(3):911-915. [PubMed: 11110963]

29. Ho VB, Corse WR, Hood MN, Rowedder AM. MRA of the thoracic vessels. Semin Ultrasound CT MR. 2003; 24(4):192-216. [PubMed: 12954004] 


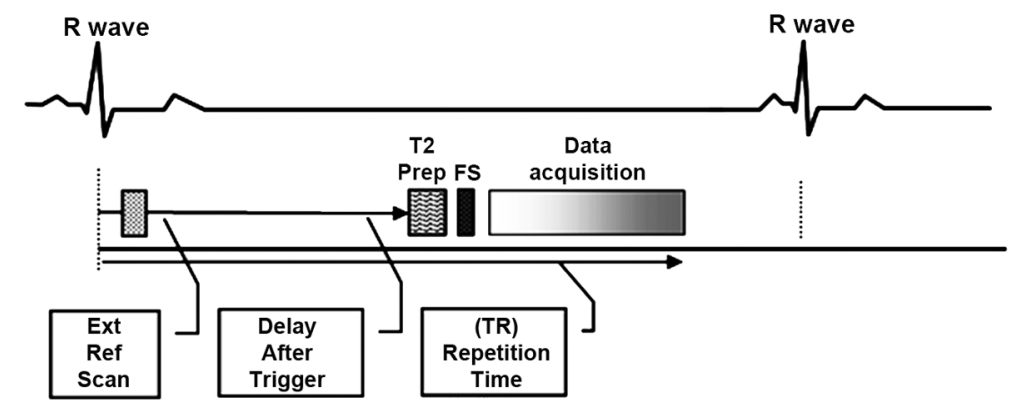

Figure 1.

Schematic of NC-MRA using ECG-triggered, T2-prepared, Fat-saturated, segmented bSSFP with the coil sensitivity and image data acquired at two different cardiac phases (early systole and mid diastole, respectively) in the same cardiac cycle within a single $\mathrm{BH}$, in order to increase the acceleration efficiency and avoid the misregistration due to varying breath hold positions. 


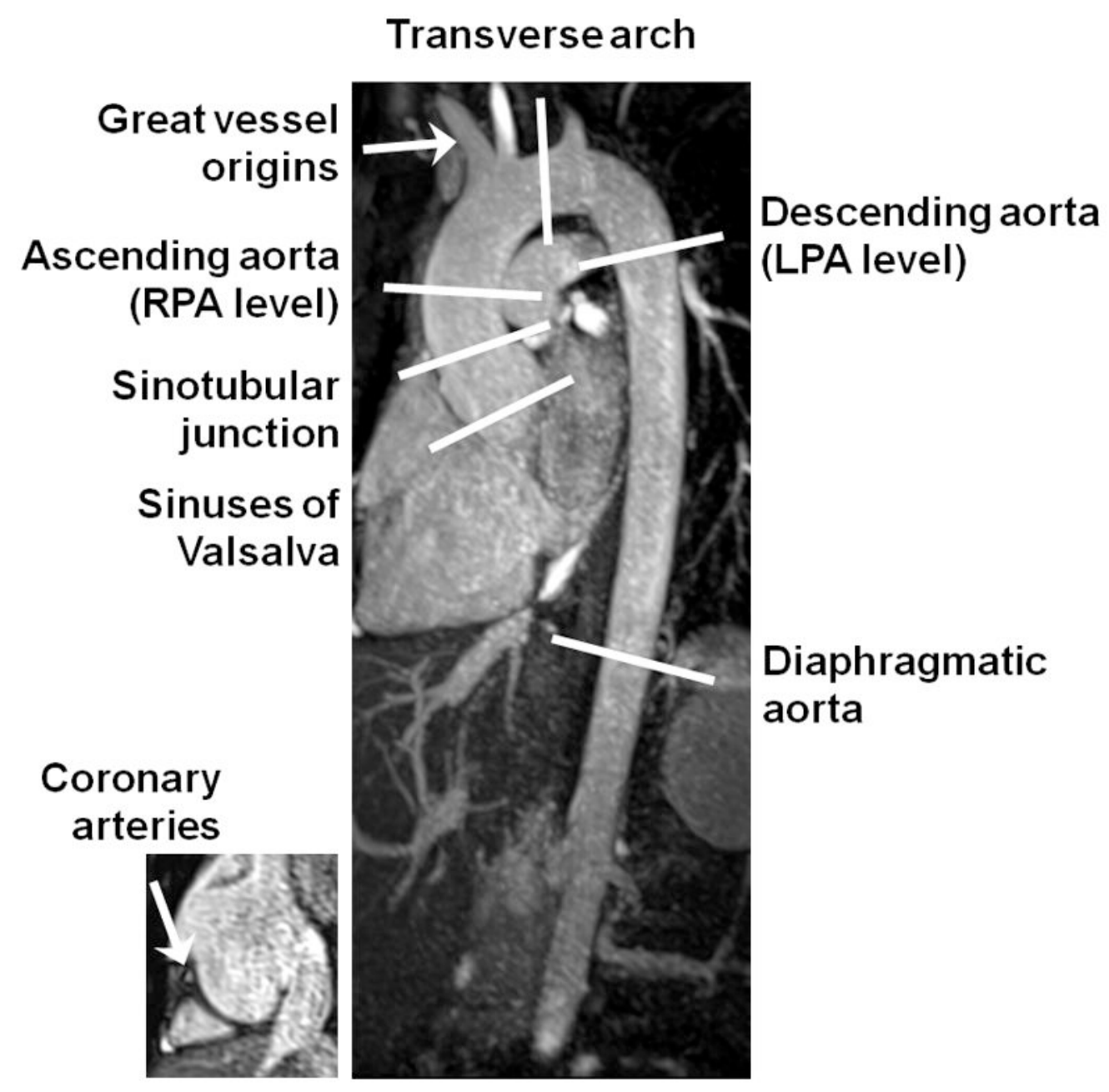

Figure 2.

Diagram of the thoracic aorta demonstrates the segments used for the evaluation of image quality and the levels used for the measurement of aortic dimensions. 


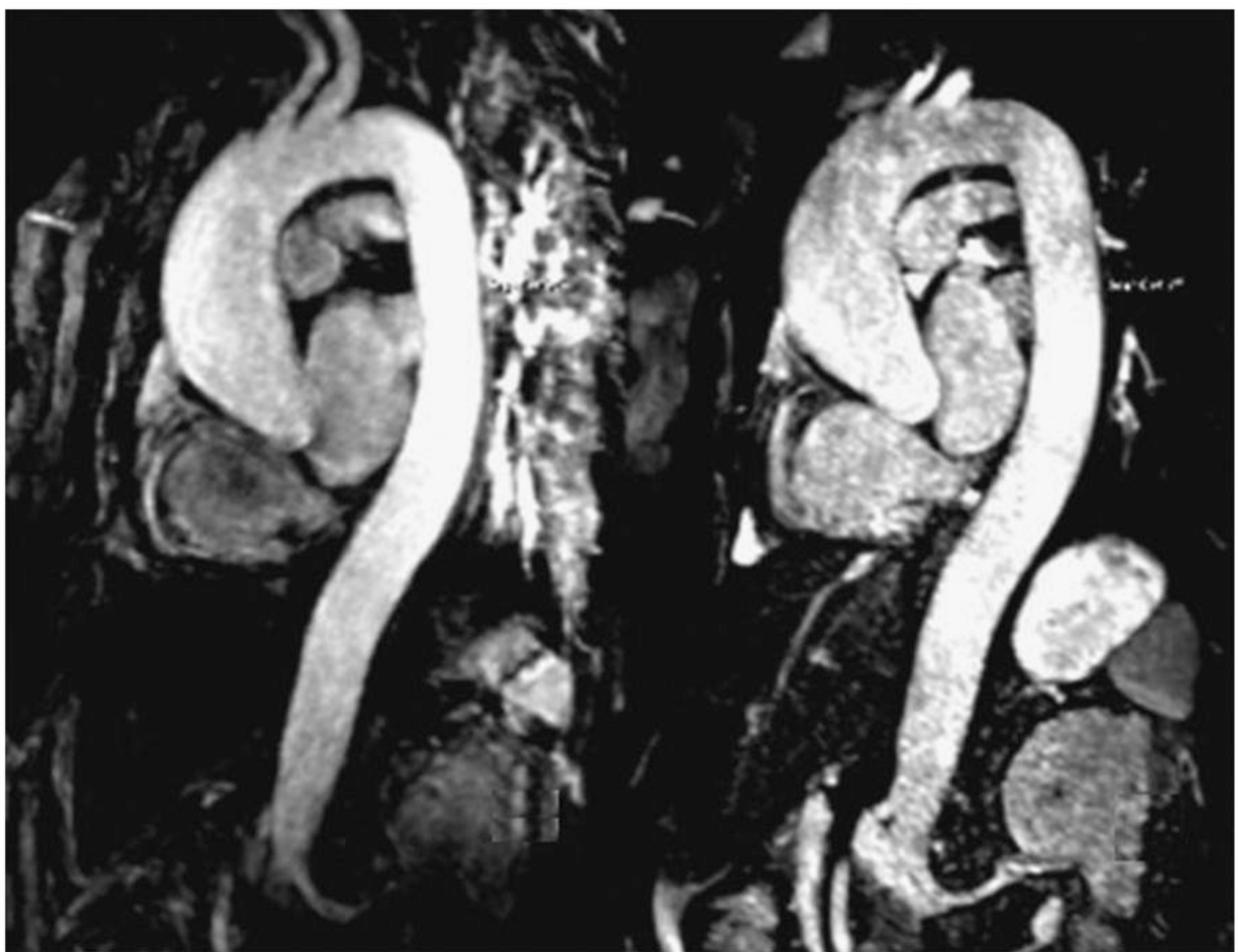

Figure 3.

MR images in a 59-year-old patient suffering from aneurysm of the aorta root obtained with NC-MRA (Right) and CE-MRA (Left) sequence. The relative merits of each technique are: CE-MRA provides superior vascular delineation due to the enhancement by contrast; whereas the single BH ECG-triggered NC-MRA is relatively free from motion artifact, so cardiac morphology is more clearly visualized, with sharper delineation of the aortic root and better assessment of coronary artery origins, due to its much shorter acquisition window (110 ms vs. 216 ms; NC-MRA vs. CE-MRA, respectively). 


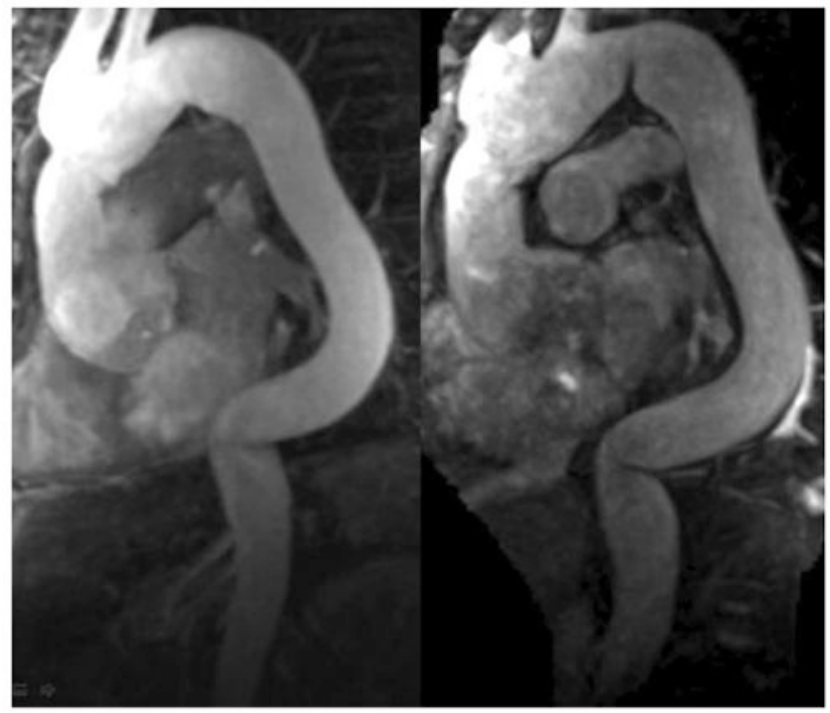

A

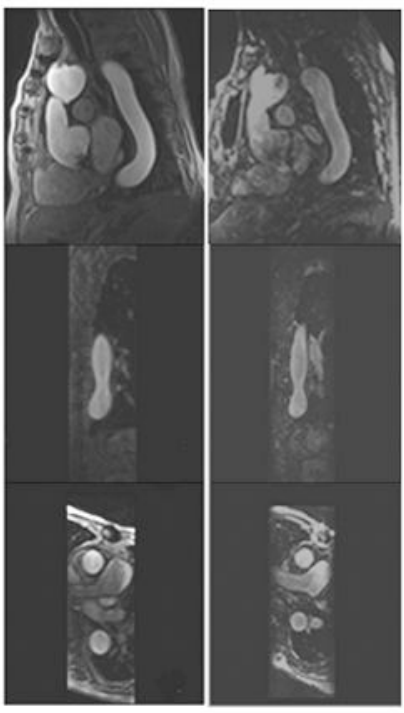

B

Figure 4.

A) MR images in a 79-year-old patient with tortuous thoracic aorta obtained with NC-MRA (Right) and CE-MRA (Left) sequence. The reconstruction planes clearly demonstrate sharper delineation of the aorta root and other aorta segments with high image quality for NC-MRA. B) Multiplanar reformatted images from NC-MRA (Right) and CE-MRA (Left) at the mid-descending aorta: sagittal, coronal and axial images. 


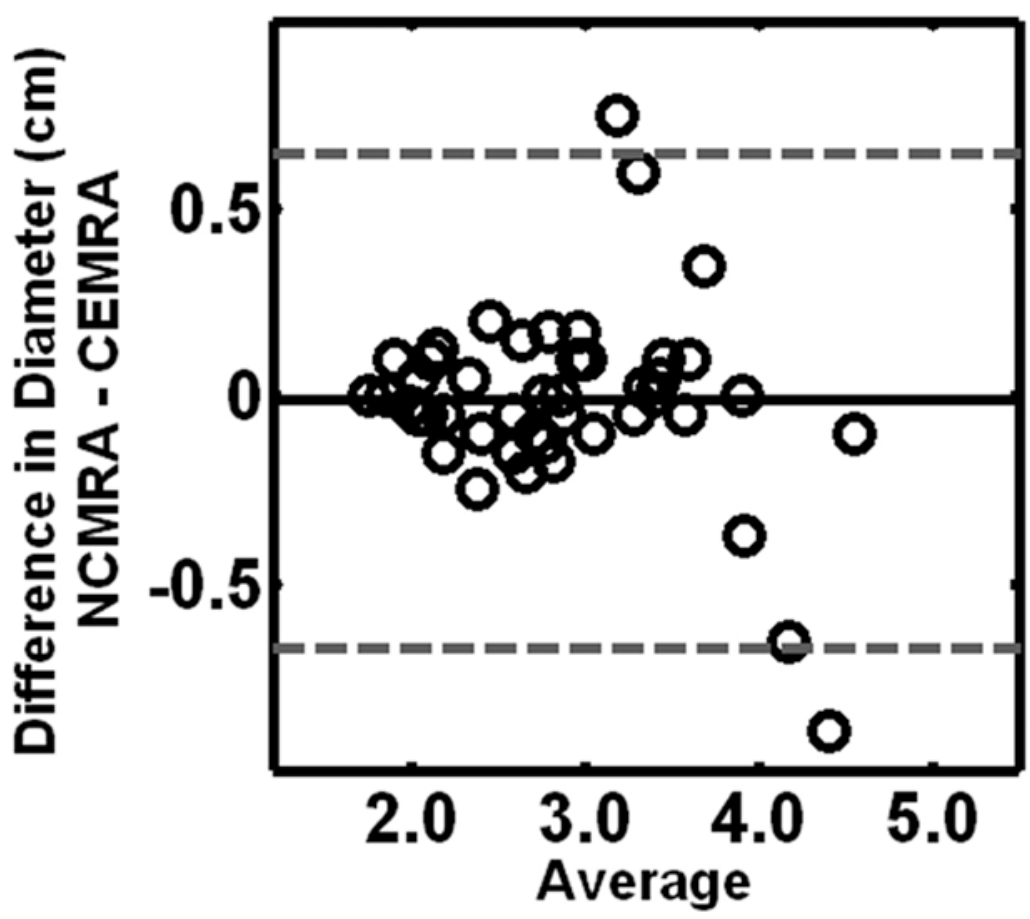

Figure 5.

Bland-Altman plot shows that overall measurements made with 3D NC-MRA and CE-MRA data sets were in good agreement with no significant difrerence in measured values. BlandAltman analysis revealed a mean measured difference (NC-MRA minus CE-MRA) in aortic diameter between the two techniques of $-0.073+/-0.144 \mathrm{~cm}$ upper and lower $95 \%$ limits of agreement $=0.210$ and -0.357 , respectively. 


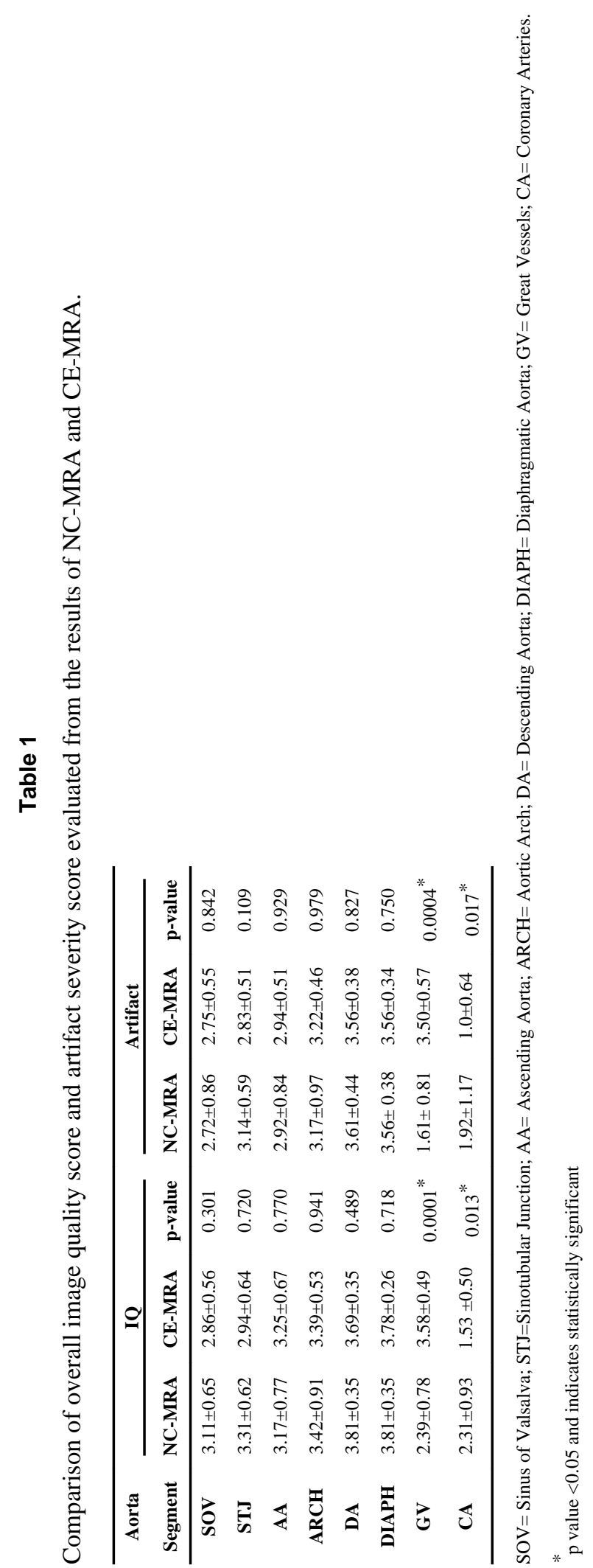

J Magn Reson Imaging. Author manuscript; available in PMC 2013 April 1. 


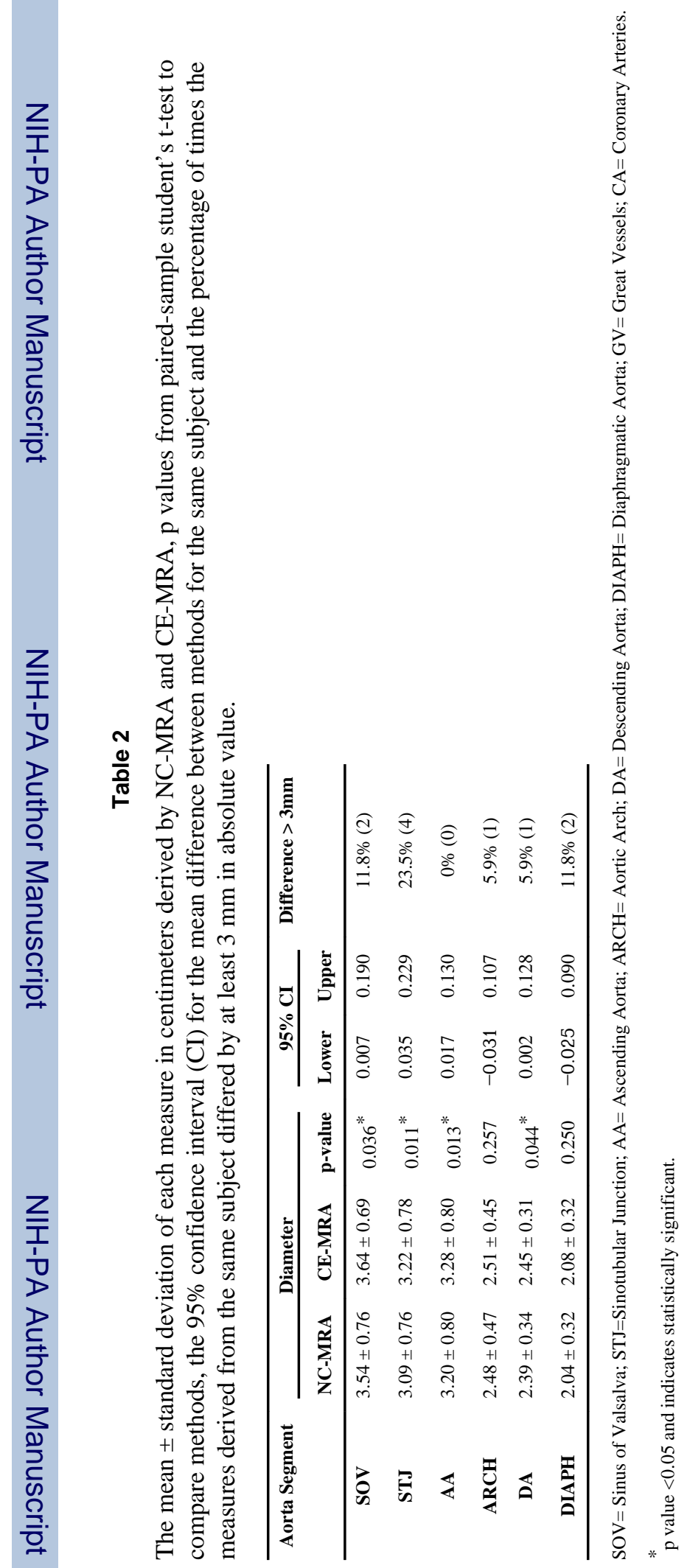

J Magn Reson Imaging. Author manuscript; available in PMC 2013 April 1. 